\title{
The large-scale anisotropy of cosmic rays observed with the partial LHAASO-KM2A array
}

\author{
Wei Gao*t \\ Institute of Frontier and Interdisciplinary Science, Shandong University, 266237 Qingdao, \\ China. \\ E-mail: w1sjzgaoweid163.com
}

\section{Wenlong Li, Chengguang Zhu}

Institute of Frontier and Interdisciplinary Science, Shandong University, 266237 Qingdao, China.

\section{Songzhan Chen, Zhe Li}

Key Laboratory of Particle Astrophysics, Institute of High Energy Physics, CAS, 100049 Beijing, China.

\section{Shuwang Cui, Peipei Zhang}

Hebei Normal University. 050024 Shijiazhuang, China.

\section{On behalf of the LHAASO Collaboration}

\begin{abstract}
The Large High Altitude Air Shower Observatory (LHAASO) is a hybrid extensive air shower array in Sichuan, China, at an altitude of 4,410 $\mathrm{m}$ above the sea level. KM2A is one of the major sub-arrays of LHAASO. It consists 5195 Electromagnetic particle Detectors (ED) and 1171 Muon Detectors (MD), covering an area of about $1.36 \mathrm{~km}^{2}$. KM2A could detect cosmic rays from 10 $\mathrm{TeV}$ to $100 \mathrm{PeV}$ with good element discrimination ability. It will enrich the measurement of the large-scale anisotropy of cosmic rays. This will throw light on the origin of the large-scale anisotropy of cosmic rays, as well as on the cosmic ray origin. The construction of detectors is underway and a part of KM2A array has been operated with 33 EDs since February 2018. In this work, we report the preliminary observation of the large-scale anisotropy using the data collected by the partial KM2A array during 2018.
\end{abstract}

36th International Cosmic Ray Conference -ICRC2019-

July 24th - August 1st, 2019

Madison, WI, U.S.A.

\footnotetext{
* Speaker.

${ }^{\dagger}$ This work is supported by the Chinese Postdoctoral Science Foundation (No. 2019M652357), the Natural Sciences Foundation of China (No. U1831129), National Key R\&D Program of China (No. 2018YFA0404201), and the Natural Sciences Foundation of China (No. 11575203 and 11635011).
} 


\section{Introduction}

Cosmic rays, mainly atomic nuclei from outer space, have been observed by a number of spaced-based detectors and ground-based arrays. Many important progresses have been achieved. From $10^{9} \mathrm{eV}$ up to beyond $10^{20} \mathrm{eV}$, cosmic rays follow a steeply falling power-law spectrum. From $\mathrm{PeV}$ to $\mathrm{EeV}$, the cosmic-ray spectrum has three prominent features. Around $4 \mathrm{PeV}$, known as the "knee", the index changes from 2.7 to 3.1 [四]. The index of the "second knee" []] at about $0.4 \mathrm{EeV}$ becomes harder to 3.3. With the increase of the energy, the spectrum becomes softer around $4 \mathrm{EeV}$, which is known as the "ankle". It's believed that cosmic rays with energies below the "knee" may originate within Galactic objects. The "knee" may be due to the flux cutoff of light component of cosmic rays, while the "second knee" may be caused by the escape of heavy components. The "ankle" may reflect the transition of cosmic rays from galactic to extragalactic origin. For galactic cosmic rays, particles are assumed to be accelerated in sources, most likely in shocks from supernova explosions. However, the cosmic-ray origin is still mystery. To measure the large-scale anisotropy (LSA) of cosmic rays is an important way to understand the cosmic-ray acceleration and propagation.

From $50 \mathrm{GeV}$ to $8 \mathrm{EeV}$, many experiments have detected the cosmic-ray anisotropy and ac-

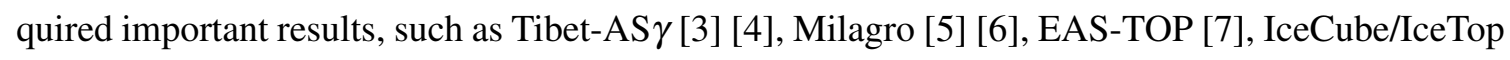

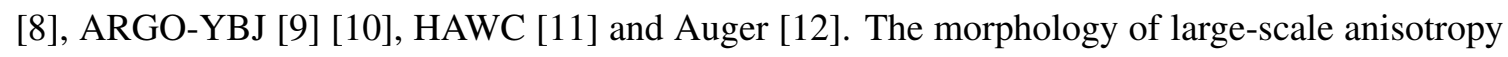
and the corresponding amplitude are energy dependent. From sub-TeV to tens of $\mathrm{TeV}$, the morphology of anisotropy is dominated by two large-scale features, the excess and deficit features known as "Tail-in" and "loss-cone" separately both in northern and southern hemispheres. Recently, the

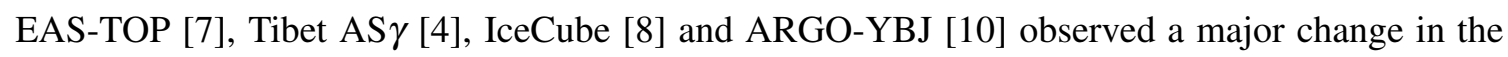
morphology above hundreds of TeV. "Tail-in" and "Loss-cone" are fading away while a wide range of excess including the direction of the Galactic center appears. That perhaps indicates a change of the dominant origin of cosmic rays. At ultrahigh energy, the Auger collaboration detected the large-scale anisotropy at an energy above $8 \mathrm{EeV}$ [ए2]. Its magnitude and direction of the anisotropy support the hypothesis of an extragalactic origin for the highest-energy cosmic rays, rather than sources within the galaxy. It's worth to note that the ARGO-YBJ collaboration tried to study the anisotropy dependence on the nature of the primaries. Limited by the ability of particle identification, the ARGO collaboration reported a preliminary result [[13]. Studying the anisotropy in different energy ranges and the anisotropy of different elemental composition of cosmic rays are helpful for us to understand the origin and propagation of cosmic rays.

The Large High Altitude Air Shower Observatory (LHAASO) [ㅍ] is a new generation hybrid extensive air shower (EAS) array, which could detect cosmic rays from hundreds $\mathrm{GeV}$ up to EeV. Such advantages allow for observations of the anisotropy in a very wide energy range. By the way, the good particle discrimination ability could provide measurements, for the first time, of the cosmic-ray anisotropy across the knee separately for light and heavy primary masses. That will enrich measurements of the large-scale anisotropy of cosmic rays. As LHAASO is under construction, the partial array has already started operation. In this paper, a preliminary measurement of large-scale anisotropy is introduced with data accumulated by the partial array. 


\section{The LHAASO experiment and the Data}

\subsection{The LHAASO experiment}

The Large High Altitude Air Shower Observatory, which located in Daocheng, Sichuan province of China, at an altitude of 4,410 a.s.l., is aimed to explore the origin of high-energy cosmic rays. LHAASO will be devoted to studying a wide range of fundamental issues in cosmic ray and astroparticle physics, such as the gamma-ray astronomy, energy spectrum of individual cosmic ray elements, large-scale anisotropy of cosmic rays, and solar physics. As shown in Figure M, LHAASO contains three arrays with different types of detectors. One kilometer array (KM2A), covering about $1.36 \mathrm{~km}^{2}$ area, is consisted of 5195 Electromagnetic particle Detectors (EDs) and 1171 Muon Detectors (MDs). KM2A has good identifying ability for gamma rays and cosmic rays from $10 \mathrm{TeV}$ to $100 \mathrm{PeV}$ with EDs and MDs to detect the electromagnetic particles and muons of EAS, separately. Located in the center of LHAASO, the Water Cherenkov Detector Array (WCDA), which covering about $78,000 \mathrm{~m}^{2}$ area and constituted by 3120 detector units is divided into three separate arrays. The WCDA detects Cherenkov light generated by shower secondary particles in water with a very high background rejection power and a good angular resolution from $100 \mathrm{GeV}$ to 20 TeV. The Wide Field of View Cherenkov Telescope Array (WFCTA), composed of 12 wide-fieldof-view Cherenkov/fluorescence telescopes, could detect showers above $100 \mathrm{PeV}$. Each telescope consists of a spherical light collector of about $4.7 \mathrm{~m}^{2}$ and focal plane camera of $32 \times 32$ pixels with a pixel size of 0.5 degree. The WFCTA has two work modes to detect the Cherenkov and fluorescence light generated by shower secondary particles in air respectively. The deployment of detectors is under construction and progressing smoothly. The whole array of LHAASO is expected to be completed by the end of 2020. The deployed detectors of LHAASO have already been in operation.

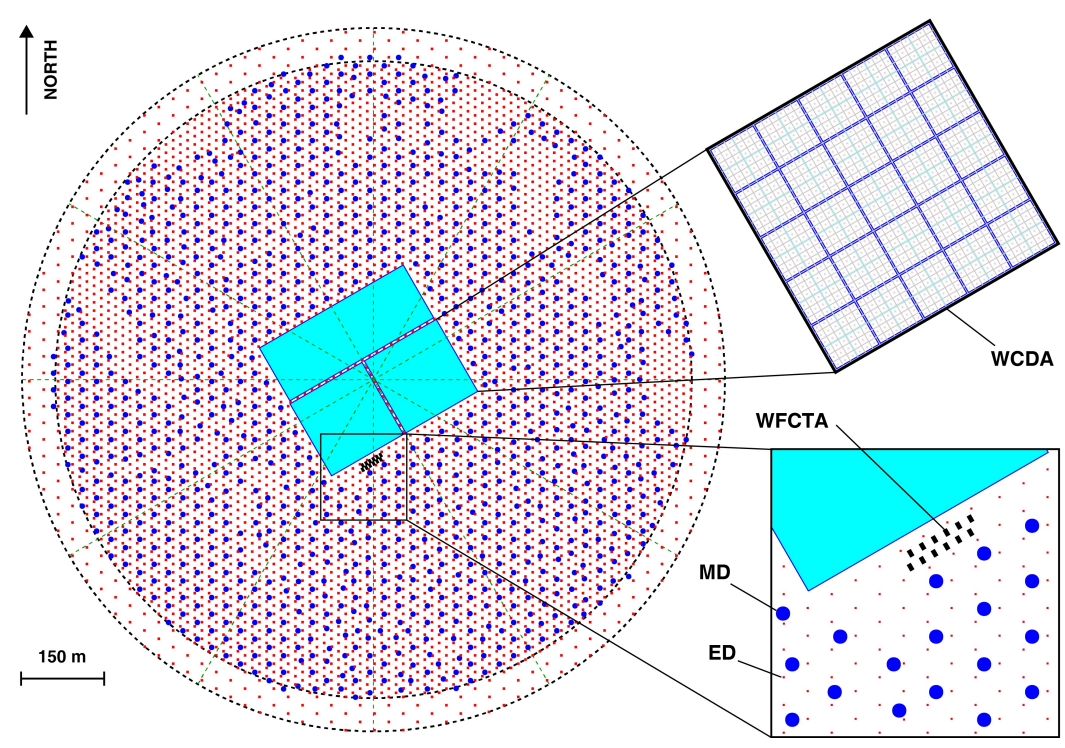

Figure 1: Layout of the LHAASO experiment. The insets show the details of one pond of the WCDA and of the KM2A array constituted by two over-imposed arrays of electromagnetic particle detectors (EDs) and of muon detectors (MDs). WFCTA telescopes, located at the edge of a pond, are also shown. [U4]] 


\subsection{Simulation and the Data Set}

A partial array of KM2A, consists 33 EDs, starts on operation since February, 2018. Data collected by 33 EDs during 2018 is used in this work. The event rate of 33 EDs is around $42 \mathrm{~Hz}$, shown as Figure $\square$. During the array operation, many detectors were replaced the power supply and the threshold of signals were adjusted, that cause the counting rate unstable. In this work, data selection is made as fellow cuts: (1) number of triggered ED after noise filter is not less than 5, (2) the zenith angle of reconstructed events is less than 50 degree.

The energy of cosmic rays is estimated by the Monte Carlo simulation. Five groups of dominant component elements, $\mathrm{H}, \mathrm{He}, \mathrm{CNO}, \mathrm{MgAlSi}$, and $\mathrm{Fe}$, are generated according to [प[5]]. The interaction of cosmic rays in the atmosphere is simulated by CORSIKA code v.7.6400, with the hadronic interaction model Fluka at lower energy and EPOS at higher energy. About $3.98 \times 10^{10}$ events are sampled with zenith angle distributed from $0^{\circ}$ to $70^{\circ}$ and the energy distributed from 0.1 $\mathrm{TeV}$ to $10 \mathrm{PeV}$. The detector response is simulated with the G4KM2A code based on GEANT-4 [10].

In this work, about $7.2 \times 10^{8}$ detected events are reserved after data selection. The largescale anisotropy in different energy ranges are also studied. Events are divided into three samples according to the fired ED's number with noise filtering ( $n$ filtE): $5 \leq n$ filtE $<10,10 \leq n$ filtE $<$ 20 , and $n$ filt $E \geq 20$. The event number of three samples are about $4.01 \times 10^{8}, 2.42 \times 10^{8}$ and $7.50 \times 10^{7}$, respectively. The energy distribution of these events, i.e., the fraction of events as a function of the primary energy, is shown in Figure B]. The energy of events, which $n$ filtE not less than 5 , is about $36.5 \mathrm{TeV}$ estimated with the simulation (left plot). And the estimated energy of three samples are 23.7, 46.3 and 119.2 TeV separatively (right plot).

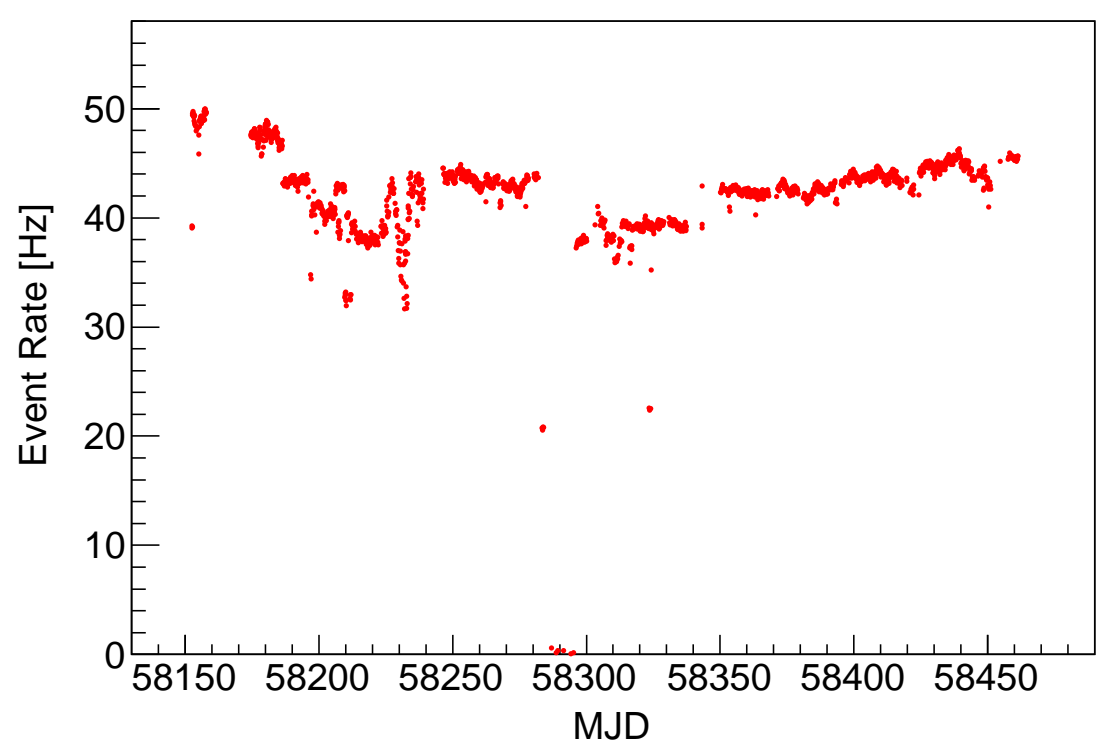

Figure 2: The event rate of 33 EDs during 2018. 

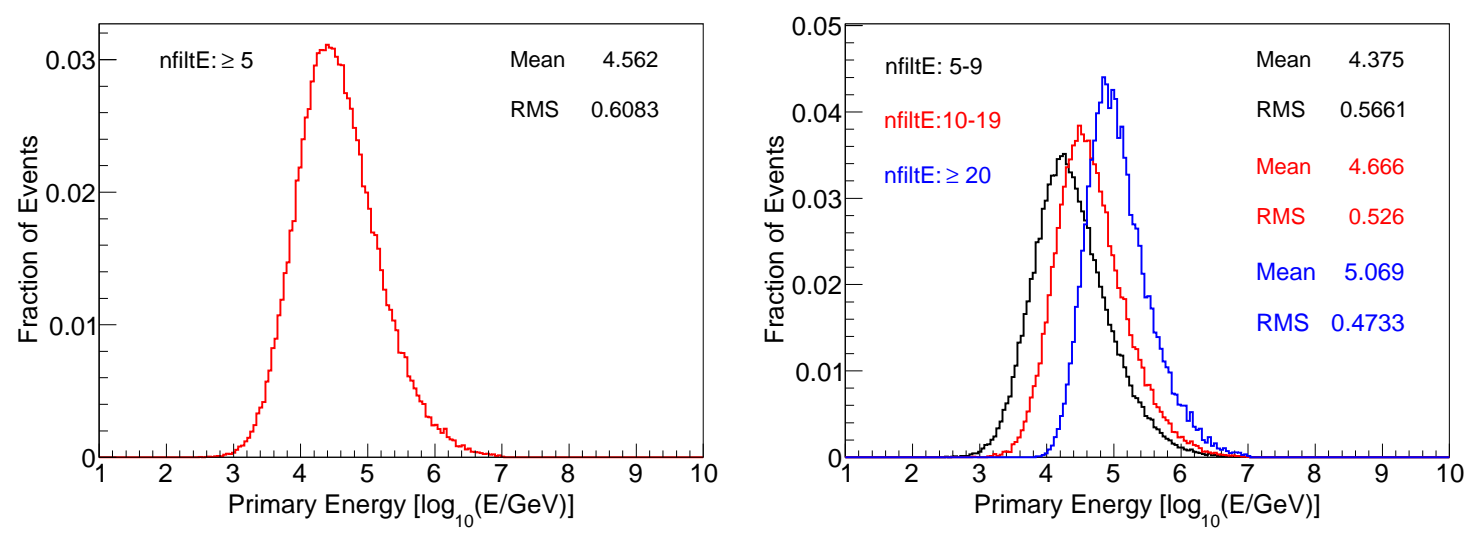

Figure 3: Fraction of events as a function of the primary energy for data samples used in this analysis, from simulation. The primary cosmic-ray composition and energy spectrum are based on [ㅍ]]. The left plot is for data sample with $n f i l t E \geq 5$, and the right plot for three $n f i l t E$ bins.

\section{Preliminary Results}

The equi-zenith angle method based on an iterative procedure [[T] is used to estimate the background of cosmic rays in this work. With this approach, the two dimensional (2D) large-scale anisotropy of cosmic rays are determined. Figure $⿴$ t shows the large-scale anisotropy detected by KM2A partial array at about $36.5 \mathrm{TeV}$. The $2 \mathrm{D}$ maps are smoothed with $30^{\circ}$ angular radius. The top plot is the significance and the middle one is the relative intensity of large-scale anisotropy. Two wide range features, known as "tail-in" and "loss-cone", are observed with significance of 10.48 $\sigma$ and $-11.37 \sigma$ separately. The bottom plot is the one dimensional (1D) projection of relative intensity on the direction of right ascension. The red symbols represent the relative intensity in sidereal time and the blacks in anti-sidereal time, that is usually used to estimate the systematic errors.

Figure $\square$ shows the 2D maps of significance (left plots) and relative intensity (right plots) of cosmic-ray anisotropy of three samples. The vanishing of "Tail-in" and "Loss-cone" are observed. As shown in Figure $\mathbb{6}$, the 1D projections of intensity on right ascension are fit with an equation of $I=1+\operatorname{amp} \cos (\alpha-\phi)$ to quantitatively estimate the evolution of the large-scale anisotropy. The left panels represent relative intensity in sidereal time, and right panels in anti-sidereal time, amplitudes in which are usually used to estimate the systematic error. It's obviously that the amplitude of large scale anisotropy is decreasing at higher energy interval. At $119.2 \mathrm{TeV}$, the amplitude of anisotropy intensity in sidereal time is smaller than that in anti-sidereal, such meaning a larger systematic uncertainty. For comparison, results reported by other experiments and this work are shown in Figure $\square$. The left and right panels are the amplitude and phase of large-scale anisotropy, respectively. The results obtained in this work generally consistent with others.

\section{Summary}

This paper reports on the measurement of the large-scale anisotropy of cosmic rays by a partial 

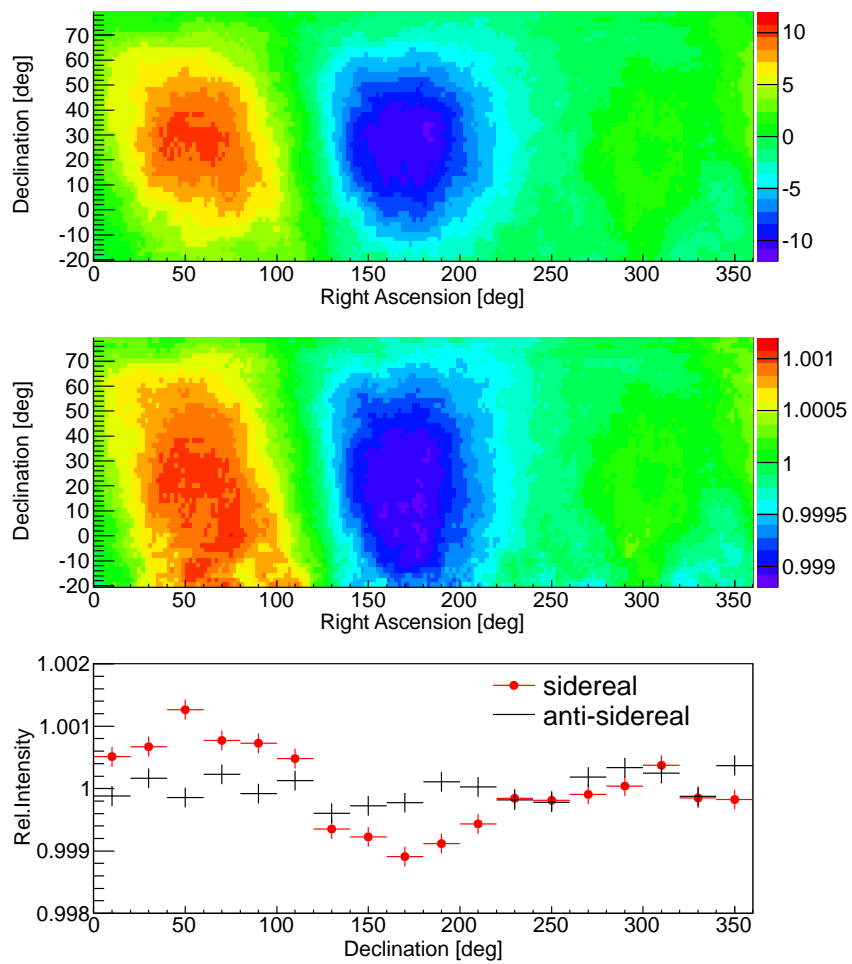

Figure 4: Large-scale anisotropy measured by KM2A partial array at about 36.5 TeV. 2D maps are smoothed with $30^{\circ}$ angular radius. The up and middle plots represent the significance and relative intensity of anisotropy separatively. The bottom plot shows the 1D profiles of relative intensity in sidereal time (red) and anti-sidereal (black) time.
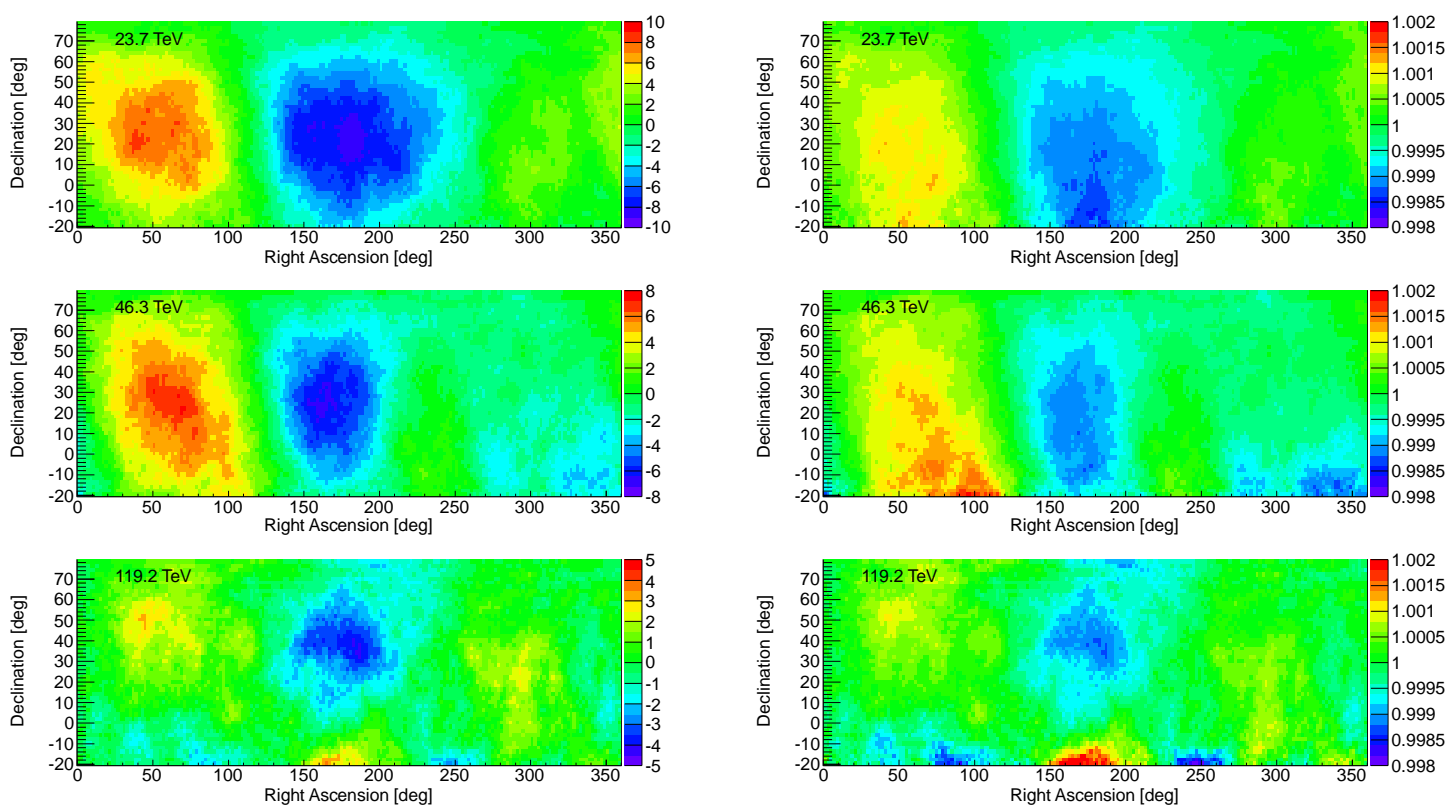

Figure 5: The 2D maps of large-scale anisotropy at $23.7 \mathrm{TeV}, 46.3 \mathrm{TeV}$ and $119.2 \mathrm{TeV}$ with $30^{\circ}$ angular radius smoothing. 

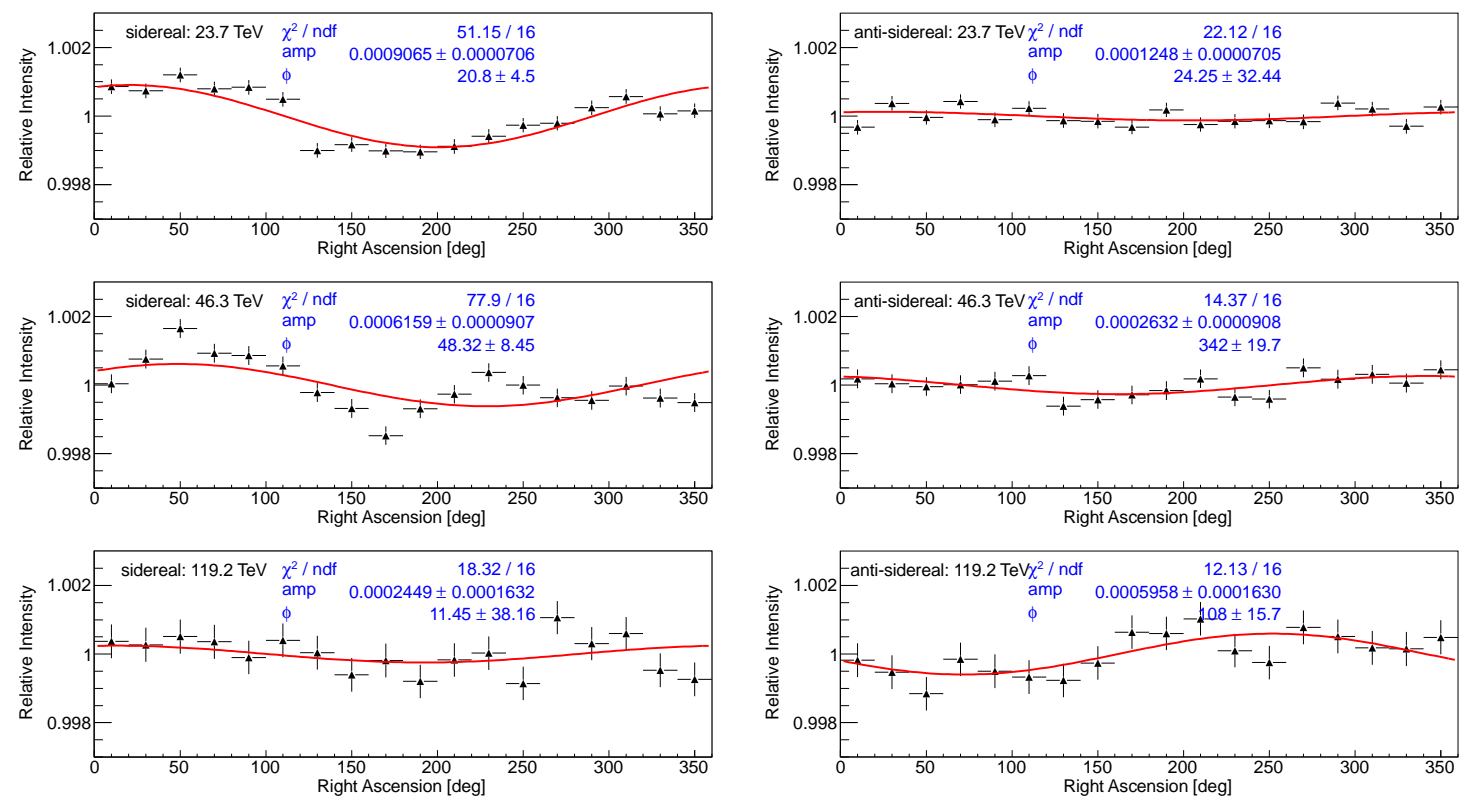

Figure 6: The 1D projections of relative intensity on right ascension in sidereal (left panels) and anti-sidereal (right panels) time at $23.7 \mathrm{TeV}, 46.3 \mathrm{TeV}$ and $119.2 \mathrm{TeV}$.
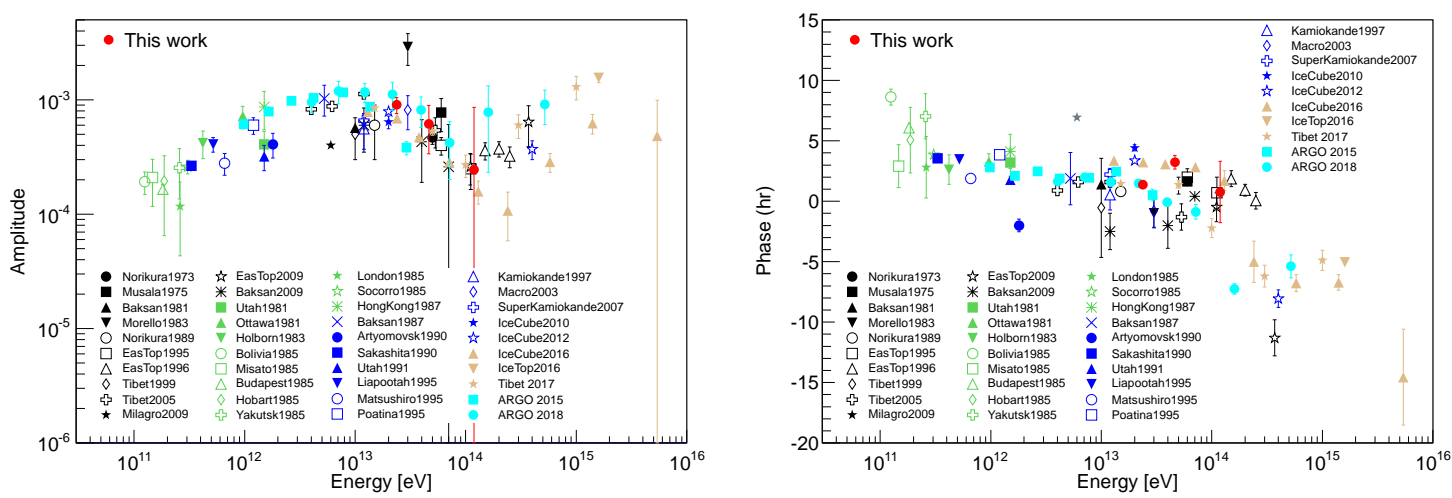

Figure 7: The amplitude (left plot) and phase (right plot) of the sidereal anisotropy measured by KM2A partial array (big red dot), as a function of the cosmic ray energy (in the units of eV), along with the results from other experiments.

array of LHAASO-KM2A. The partial array contains 33 EDs and started operation during 2018. Preliminary results obtained in this work are generally consistent with others. The features known as "Tail-in" and "Loss-cone" are observed with $10.48 \sigma$ and $-11.37 \sigma$ significance separately. Even though the limit statistics lead to a larger uncertainty, while the evolution of anisotropy at about 23.7 TeV, 46.3 TeV and 119.2 TeV energies are consistent with others. The consistent results about anisotropy are affirmations for our experiment construction.

LHAASO is under construction and progressing smoothly. The first water pool of WCDA started taking data in April, 2019. Partial array of KM2A started operation since February, 2018. With the detector deployment on going, 1/4 of the LHAASO array will be on operation soon and 
the whole array will be completed by the end of 2020. The advantages of LHAASO, such as huge statistics, wide energy range and good ability on particle identification et al., will allow more precise studies for cosmic-ray anisotropy.

\section{Acknowledgments}

This work is supported by the Chinese Postdoctoral Science Foundation (No. 2019M652357), the Natural Sciences Foundation of China (No. U1831129), National Key R\&D Program of China (No. 2018YFA0404201), and the Natural Sciences Foundation of China (No. 11575203 and 11635011).

\section{References}

[1] G. V. Kulikov et al., 1958, J. Exp. Theor. Phys., 35:635144

[2] D. J. Bird et al., 1994, ApJ, 424:491502

[3] M. Amenomori et al., 2006, Science, 314

[4] M. Amenomori et al., 2017, ApJ, 836:153

[5] A. A. Abdo et al., 2008, PRL, 101:221101

[6] A. A. Abdo et al., 2009, ApJ, 698:2121

[7] M. Aglietta et al., 2009, ApJ, 692:L130

[8] M. G. Aartsen et al., 2016, ApJ, 826:220

[9] B. Bartoli et al., 2015, ApJ, 809:90

[10] B. Bartoli et al., 2018, ApJ, 861:93

[11] A.U. Abeysekara et al., 2018, arXiv:1805.01847v2

[12] A. Aab et al., 2017, Science, 357:1266

[13] W. Gao et al., 2017, 35th ICRC, 554

[14] H. H. He, 2018, Rad. Detect. Tech. Meth., 2:7

[15] T. K. Gaisser et al., 2013, FrPhy, 8: 748

[16] S.Z. Chen et al., 2019, 36th ICRC

[17] T. L. Li et al., 2012, APh, 39-40: 144-148 